\title{
Short-term numerical avalanche forecast used operationally at Météo-France over the Alps and Pyrenees
}

\author{
Yves Durand, Gérald Giraud, Laurent Mérindol \\ Centre d'Études de la Neige, Centre National de Recherches Météorologiques, Météo-France, 38406 Saint-Martin-d'Hères Cedex, France
}

\begin{abstract}
Avalanche-hazard estimation for the present and the following days is one of the main tasks of the avalanche forecaster. For 4 years, some have used the results of a series of automatic numerical models in the Alpine massifs of France. Thee programs describe in real time the main meteorological conditions $(S A F R A \mathcal{N})$, the evolution of the snow cover (Crocus) and the resulting avalanche risks (MÉPRA) at different elevations, slopes and aspects of the massifs considered.

This paper presents the latest evolution of this automatic tool. With the new version it is now possible to provide 1 day forecasts of the state of the snow cover over the massifs of both the Alps and Pyrenees, including the main characteristics of the snowpack and an assessment of the corresponding avalanche hazards.

To achieve this result, the main changes were with SAFRAN. Two combined methods are used within the same package: adaptations of larger-scale meteorological forecasts and use of observations of analogous weather situations from the past. These two approaches are complementary especially for evaluating precipitation where the second solution has important fine-scale information while the first exhibits important local biases.

Validation of this new application was done carefully and proved the quality of the method, now used in real-time by local forecasters. We present some validation results, concerned both with forecasted precipitation fields at the scale of the massif and forecasted avalanche risks deduced from MEPRA.
\end{abstract}

\section{INTRODUCTION}

Our objective is the real-time numerical simulation of snowcover evolution and an assessment of the corresponding avalanche risks. The basic assumptions of the system are:

hourly knowledge of meteorological conditions at every computation point

climatological homogeneity of the different massifs, especially for precipitation (a map of the different massifs can be seen in Figure 4)

The current numerical chain (Giraud and others, 1994) is therefore composed of three separate models which simulate the main features of the snow cover at the massif scale $\left(\sim 500 \mathrm{~km}^{2}\right)$. Within each massif, all the parameters involved are computed for different elevations and aspects. The working scale is well suited to resolution of the different information sources used (observation network and meteorological models) and allows representation of the main structure of the snow mantle, except that due to local effects such as wind transport or different orographic forcing.

The evolution of the chain presented below concerns its ability to forecast snowpack states and avalanche risks at a lday range.

\section{BACKGROUND}

The prevailing meteorological conditions have a major influence on the evolution of the snow cover. They govern implicitly the different energy and mass budgets of every layer as well as the type of snow crystal being formed, most of the physical properties and the liquid-water content of the snow. All these quantities are very important for avalanche hazard-forecasting, one of the tasks of the French Meteorological Office (Météo-France, MF). Numerical models of the snow cover have also become efficient and relatively reliable tools; their first application is to provide a complete description of the snowpack covering the different massifs. They are widely used by those forecasting avalanche risks. The numerical simulations complement their own skills, especially in areas with sparse observations or during off-winter-season periods. The numerical products provide both a daily description of the snowpack state and its evolution in the following days.

\section{THE GURRENT SYSTEM AND ITS LIMITS}

Snow evolution is simulated by the Crocus model (Brun and others, 1989, 1992), according to the hourly meteorological parameters provided: air temperature, humidity and wind, precipitation and its phase, incoming solar and atmospheric radiation, and cloudiness. The main advantage of this model is it provides appropriate parameterizations especially for simulating the stratigraphy of the different layers and their metamorphism.

Avalanche diagnosis is performed by the MÉPRA expert model (Giraud, 1993). It estimates the main mechanical properties (e.g. shear strength, shear stress, and ram hardness) of the different layers and performs a mechanical stability analysis of the snowpack simulated by Crocus. Thus, a 
diagnosis of natural risk (comparison between shear strength and shear stress, with a specific analysis in the case of wet snow) and accidental risks (search of slabs including weak layers and influence of an overloading) is obtained for all profiles with different slopes with an indication of the likely underlying processes.

Prior to the above computations for the snow, the meteorological conditions are estimated (we shall say "analysed") by $S A F R A \mathcal{N}$ (Durand and others, 1993). The purpose of the analysis process is to provide the requested atmospheric data for all massifs, by elevation bands of 200 or $300 \mathrm{~m}$ and for the different major slope aspects (north, east, south, west and flat areas) at hourly intervals. This goal is achieved by treating all the available information: observations obtained by different networks and meteorological numerical forecasts of the ARPEGE MF model (Courtier and others, 1991). SAFRAN operates as a two-step process. In the first step, $A R P \grave{E} G E$ computes a preliminary estimate of the desired quantities (commonly called "guess field" in the literature) using suitable down-scaling operators (Durand and others, 1993) in order to take into consideration the initial ARPEGE smoothed orography compared to our finer working scale. Afterwards these preliminary estimated fields are modified with available observations. Only for precipitation, ARPĖGEs outputs are replaced with specific climatological fields selected according to different typical weather conditions deduced from the shape of the $500 \mathrm{hPa}$ geopotential field; 7 possible guesses in the Alps and 9 in the Pyrenees are used.

The three models are run daily; allowing us to follow the snow-cover state some hours after the last observations had been made. However, it was soon confirmed that knowledge only of the previous snow state was insufficient, especially when the meteorological conditions were evolving rapidly. We thus had to consider a "forecasting-working" mode covering the following day and providing a forecasted snowcover state to the avalanche forecaster. In addition, the system to be developed has to be time-consistent with its own "analysis mode" and coherent with the meteorological forecast on a larger scale, which is also used by the forecasters. All these reasons exclude any local solution disconnected from the numerical models provided by MF.

\section{AVAILABLE INFORMATION}

\section{We first identified two main sources of information:}

The ARPĖGE model, mentioned above, with its horizontal resolution of about $30 \mathrm{~km}$. As previously stated, its direct use is inadequate at our working scale; its own orography cannot represent the large Alpine valleys. Nevertheless, it is well able to simulate the synoptic perturbations which will affect the Alpine or Pyrenean chains, and it equally provides a fair estimate of the meteorological parameters in the free atmosphere. Hence the model carries a set of averaged field quantities which must be down-scaled at the massif scale.

The "nearest" meteorological conditions of the past. This method had already been successfully used and tested (Duband, 1981; Navarre, 1980). Its purpose is to find a situation in the past which closely resembles the current one by way of "minimizing a distance" between these two situations. The search is generally performed over a large geographical area in order to optimize the occurrence of similar meteorological patterns.

\section{GUSTOMIZATION OF AVAILABLE INFORMATION}

All forecasting techniques are based on $S A F R A \mathcal{N}$ which has to run without its daily observations but using the proposed information. SAFRAN must provide Crocus and MEPRA with their necessary meteorological hourly parameters (Durand and others, 1993). This purpose is achieved by continuing the analysis mode every morning (from 6 UTC the previous day to 6 UTC the current day) with a forecasting mode finishing at 6 UTC the following day. All results have to be available about 12 UTC on the current day. The proposed solution is based on customization and a combination of the two previously mentioned methods and involves the following treatments.

\section{Large-scale ARPĖGE fields}

The main task consists of a down-scaled adaptation of the outputs of the ARPÉEE model through the different operators of which an earlier version has been described by Durand and others (1993). These operators are generally only used to build the guess fields in the analysis mode. Some other operators, suitable for precipitation, were also developed. Contrary to the analysis mode, the medium-range forecasted precipitation of the ARPE $G E$ model is used in the $S A F R A \mathcal{N}$ forecasting mode.

\section{Analogous situations}

It consists of searching for the nearest-neighbour meteorological situations, beginning with the summer of 1981 . We look for a limited set of meteorological situations that resemble the ARPEGE forecast for the coming day. We generally keep the ten "closest" past situations (identified by their dates) in order to perform, if necessary, a second level of research based on the neighbouring and $A R P E G E$-forecasted precipitation patterns. The search is performed by comparing these forecasts with past meteorological fields obtained from the European Center for Medium-Range Weather Forecasts (ECMWF, 1991) analyses. To use such archives is most convenient but limits the search to the starting date of this Center, despite the fact that many previous observation sets are available for the massifs. We are also certain that a 15 year history is not sufficient to identify high-quality neighbouring situations, particularly if we consider the short return period of some important phenomena.

The "distance" used to compare two meteorological situations is based on four fields: geopotential at 500 and $700 \mathrm{hPa}$, temperature and an estimate of the temperature advection (close to the temperature temporal tendency) at $700 \mathrm{hPa}$. Various operators are then applied in order to compute this mathematical distance; they consider both the field and its first and second spatial derivatives. However, compared with the areas treated in the above-mentioned references, the geographical search area is limited (Alps: $15000 \mathrm{~km}^{2}$ or Pyrenees $10000 \mathrm{~km}^{2}$ ), a fact that can jeopardize recognition of some synoptic patterns. So this distance is computed as a function of time and integrated over 30 hours of forecast over the same geographical area in order to compensate for its smallness by a temporal dimen- 
sion. A supplementary term takes into account the forecasted and archived precipitation.

The result obtained, called hereafter the "full distance", is a number whose low values identify best neighbouring dates. It characterizes both the meteorological similarity between two dates (called the "similitude distance") and the quality of the ARPEGE forecasts compared to the ECMWF analysed fields ("forecasting distance"). We can evaluate an average value of this last uncertainty by comparing two sets of ARPלेGE forecasts for the same day. The first set is composed of 0 and 6 hour range forecasts and is of good quality, and will be our reference, while the second is composed of medium-term forecasts (from a 12-30 hour range) with a larger error that we want to evaluate. To achieve that, we compute the averaged forecasting distance between these two sets over about 2000 days. The resulting value of about 20.000 (composite distance unit, integrating all the involved variables) illustrates the inner errors of the forecast fields and can be compared with the full-distance values plotted in Figure 1. This figure shows the decrease of the averaged full distance (plotted as histograms with the scale on the left vertical axis) when we use a history search interval of 1-15 years. As mentioned, these full-distance values include both the discrepancy between the meteorological patterns ("similitude distance") and the forecasting error ("forecasting distance", estimated at 20.000), these two parts are a priori uncorrelated. The number of computed combinations for each histogram class is plotted as solid black squares on the left (in thousands). We must observe that every indication of the magnitude of the distance is dependent on its mathematical formulation (not expressed here) and therefore must be considered in a relative way. As a result of this study, we are working to increase our history period with both fields and data.

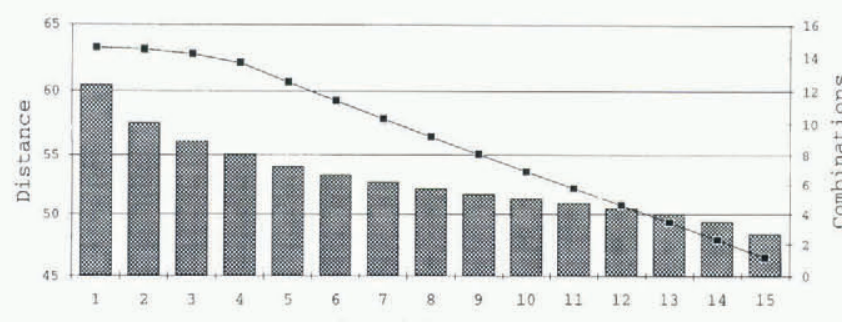

Search in years

\begin{abstract}
Fig. 1. Bar chart showing the decrease of the averaged minimization distance (in thousands) vs the number of years used in the search. It illustrates the impact of a larger sample of analogous situations in the search which leads to a closer solution characterized by a smaller distance. The magnitude of the distance (plotted on the $y$ axis) is dependent on the mathematical formulation used for the distance function and must be seen here in a relative way. The number of averaged cases in each bar is plotted (in hundreds) on the vertical right $y$ axis and decreases as the history become more important (fewer possible combinations).
\end{abstract}

There is no seasonal limitation in the search; for a given day the analogous situation is chosen within the complete set of search situations of the past without any constraint on the dates. In order to understand better how the search was carried out by the system, Figure 2 shows a histogram of the distribution of the time deviation (in months) between about 5000 reference situations and their analogous dates.

The "0" class corresponds to cases where, for a given date, the analogous situation was in the same month and the other classes refer to deviations from 1 to 6 months before or after the reference month. We can see that, by itself, the system favours the month and surrounding ones of the reference situation, but the search can have a larger deviation when for example the analogous situation of an autumn reference situation is chosen in spring.

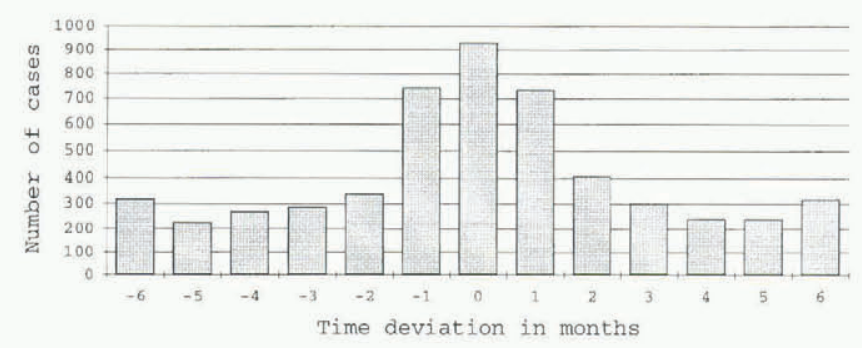

Fig. 2. Histogram of the distribution of the seasonal time deviation (expressed in months) between a date and its analogue from a sample of 5028 searches with a history of 12-15 years). The values are computed with regard to the month of the reference date and the different classes illustrate the deviation with the corresponding analogue date (independently of the year). As we can select any analogue date over the year, the values vary between -6 and +5 . The classes +6 and -6 are the same and generally illustratee cross-selections between spring and autumn.

\title{
PROPOSED METHODOLOGY
}

After experimentation, it was found that only ARPE $G E$ forecasts (after scale adaptation) provided the best solution for estimating all the fields considered except precipitation. This result has been given by Durand and others (1995) and applies over the Alps and the Pyrenees. We shall illustrate it briefly by taking some examples from two well-instrumented mountain sites: Col de Porte Chartreuse massif, $1340 \mathrm{~m}$, flat) and Col du Lac Blanc (GrandesRousses massif, $2700 \mathrm{~m}$, north). The correlation coefficients for the different analysed and forecasted parameters, compared to observations, are shown in Table 1 . They illustrate the overall quality of the forecasted fields. For temperatures

\begin{abstract}
Table 1. Correlation coefficient of different analysed and forecasted fields at an hourly time-step compared to observations (not taken in the analysis schemes) at two mountainous instrumented sites: Col de Porte (Chartreuse, $1340 \mathrm{~m}$, flat) and Col du Lac Blanc (Grandes-Rousses, $2700 \mathrm{~m}$, flat) during the 1994-95 season
\end{abstract}

Locations

Parameters / fields
Col de Porte (365 days) Coldu Lac Blanc (175 days)

$$
\begin{array}{lllllllll}
A & G & S & P & A & G & S & P
\end{array}
$$

Air temperature

Wind speed

Relative humidity

IR radiation

Global solar radiation

Cloudiness

Radiative budget

$\begin{array}{lccccccc}0.98 & 0.95 & 0.78 & 0.95 & 0.97 & 0.96 & 0.83 & 0.95 \\ 0.61 & 0.56 & 0.41 & 0.55 & 0.62 & 0.68 & 0.55 & 0.68 \\ 0.73 & 0.59 & 0.42 & 0.55 & 0.67 & 0.55 & 0.51 & 0.58 \\ 0.70 & - & 0.43 & 0.56 & 0.81 & - & 0.53 & 0.67 \\ 0.91 & 0.87 & 0.84 & 0.86 & 0.95 & 0.91 & 0.90 & 0.88 \\ 0.51 & 0.36 & 0.38 & 0.34 & 0.55 & 0.31 & 0.36 & 0.41 \\ 0.84 & - & 0.72 & 0.74 & 0.89 & - & 0.76 & 0.78\end{array}$



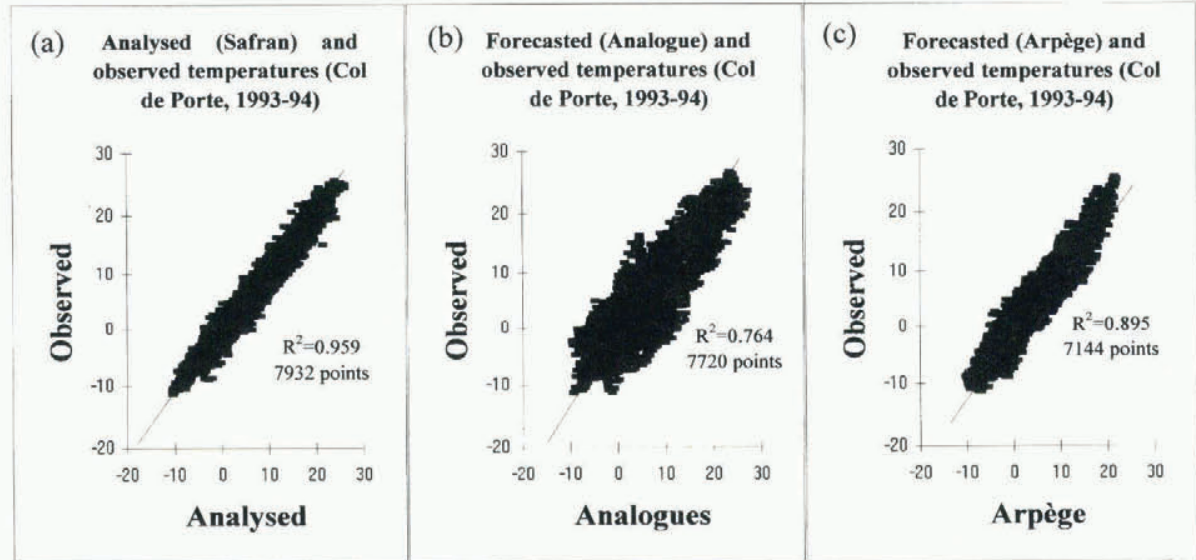

Fig. 3. Hourly analysed and forecasted temperatures compared to observations at Col de Porte (Chartreuse massif, 1340 m, flat)
during the 1993-94 season with indication of the $R^{2}$ score and number of points. Panel (a) represents the daily SAFRAN
analyses, while (b) showes the analogue-method forecasted results and (c) the ARPEGE ones.

at Col de Porte, the performance of the models are also illustrated in Figure 3. Panel (a) shows, as a reference, the reconstitution by the analysis mode (with all observations except those at the site) over the 1993-94 season with a success score $R^{2}$ (square correlation) of 0.959 ; panel (b) gives, for the same location at the same dates, the success score of the "analogue" method for which $R^{2}$ is only 0.764; panel (c) shows the ARPËGE (1 day forecast) score whose $R^{2}$ is 0.895 .

After these initial conclusions, which assumed the superiority of the simple use of the down-scaling method for all fields except precipitation, ensuing developments in this paper will be concerned only with precipitation which will also be treated by the "analogue" method.

\section{ONE-DAY RAINFALL FORECAST}

We verified (and shall discuss later) that for rainfall the "analogue" method provides information that must be taken into account. This finding seems compatible with the idea that use of the observations of the analogous situations can add a realistic variability to the raw and smoothed ARPE $G E$ rainfall fields by modulating horizontal gradients between massifs; blocking events or limited rainy areas are often observed over the Alps and Pyrenees. The ARPEGE fields have the great advantage of being implicitly consistent with the whole forecasting system of MF and not exhibiting time dephasing as the "analogues" can do. This last problem is due to the fact that one cannot be assured at all that the analogous situation for the current day, beginning at 6 UTC in the morning and finishing the following day at 6 UTC, is a previous situation starting and finishing at these same times. Some experiments (not shown here) proved that the averaged full distance values of Figure 1 (in fact its similitude part) dropped when the search was performed in steps of 6 hours instead of 24 hours, as is currently done. Nevertheless, this assumption must be made, because all available rainfall observations are 24 hour data covering this (6 UTC-6 UTC) time period and our purpose is to use these data in for an automatic analysis.

We finally deduced, from the studies described in the next section, that the procedure suitable for precipitation was a combination of the two methods presented previously, by trying to keep all their respective qualities and produce a systematically better skill score.

\section{QUALITIES OF ARPÈGE FORECASTS AND ANA- LOGOUS SITUATIONS FOR PRECIPITATION}

We began by studying the intrinsic quality of the two systems both in the Alps, with a set of about 3 years of verifica-

Table 2. Verification of 24 hour forecasted precipitation by different methods compared to reference analysis. Averaged values over Alps (c) (816 cases) and Pyrenees $(f)$ (326 cases) are shown as well as the best ( $a, d)$ and worst massifs ( $b, e)$

\begin{tabular}{|c|c|c|c|c|c|c|c|c|c|c|c|c|c|c|c|c|}
\hline \multirow{2}{*}{$\begin{array}{l}\text { Operator } \\
\text { Massifs / fields }\end{array}$} & \multicolumn{5}{|c|}{ Daily mean value } & \multicolumn{4}{|c|}{ Correlation } & \multicolumn{4}{|c|}{$2 \mathrm{cl}$. Rousseau } & \multicolumn{3}{|c|}{$5 \mathrm{cl}$. Rousseau } \\
\hline & $A$ & $S$ & $P$ & MI & M2 & $A / S$ & $A / P$ & $A / M 1$ & $A / M 2$ & $A / S$ & $A / P$ & $A / M 1$ & $A / M 2$ & $A / S$ & $A / P$ & $A / M S$ \\
\hline \multicolumn{17}{|l|}{ ALPS } \\
\hline Chablais (a) & 5.2 & 5.3 & 5.8 & 5.5 & 5.8 & 0.62 & 0.76 & 0.79 & 0.79 & 0.47 & 0.55 & 0.42 & 0.60 & 0.36 & 0.42 & 0.46 \\
\hline Queyras (b) & 3.1 & 3.0 & 6.6 & 3.3 & 2.9 & 0.43 & 0.44 & 0.50 & 0.50 & 0.50 & 0.24 & 0.20 & 0.52 & 0.31 & 0.14 & 0.30 \\
\hline All Alps (c) & 4.2 & 4.1 & 7.2 & 4.5 & 4.5 & 0.55 & 0.56 & 0.67 & 0.67 & 0.48 & 0.37 & 0.30 & 0.53 & 0.32 & 0.25 & 0.36 \\
\hline \multicolumn{17}{|l|}{ PYRENEES } \\
\hline Aspe-Ossau (d) & 6.2 & 5.2 & 6.4 & 6.2 & 6.4 & 0.39 & 0.69 & 0.69 & 0.69 & 0.46 & 0.51 & 0.44 & 0.61 & 0.28 & 0.32 & 0.39 \\
\hline Luchonnais (e) & 4.4 & 3.8 & 6.0 & 4.4 & 4.6 & 0.27 & 0.44 & 0.46 & 0.48 & 0.39 & 0.36 & 0.29 & 0.51 & 0.19 & 0.21 & 0.29 \\
\hline All Pyrenees (f) & 5.3 & 4.5 & 7.1 & 5.4 & 5.4 & 0.35 & 0.56 & 0.59 & 0.60 & 0.40 & 0.35 & 0.25 & 0.52 & 0.24 & 0.24 & 0.34 \\
\hline
\end{tabular}



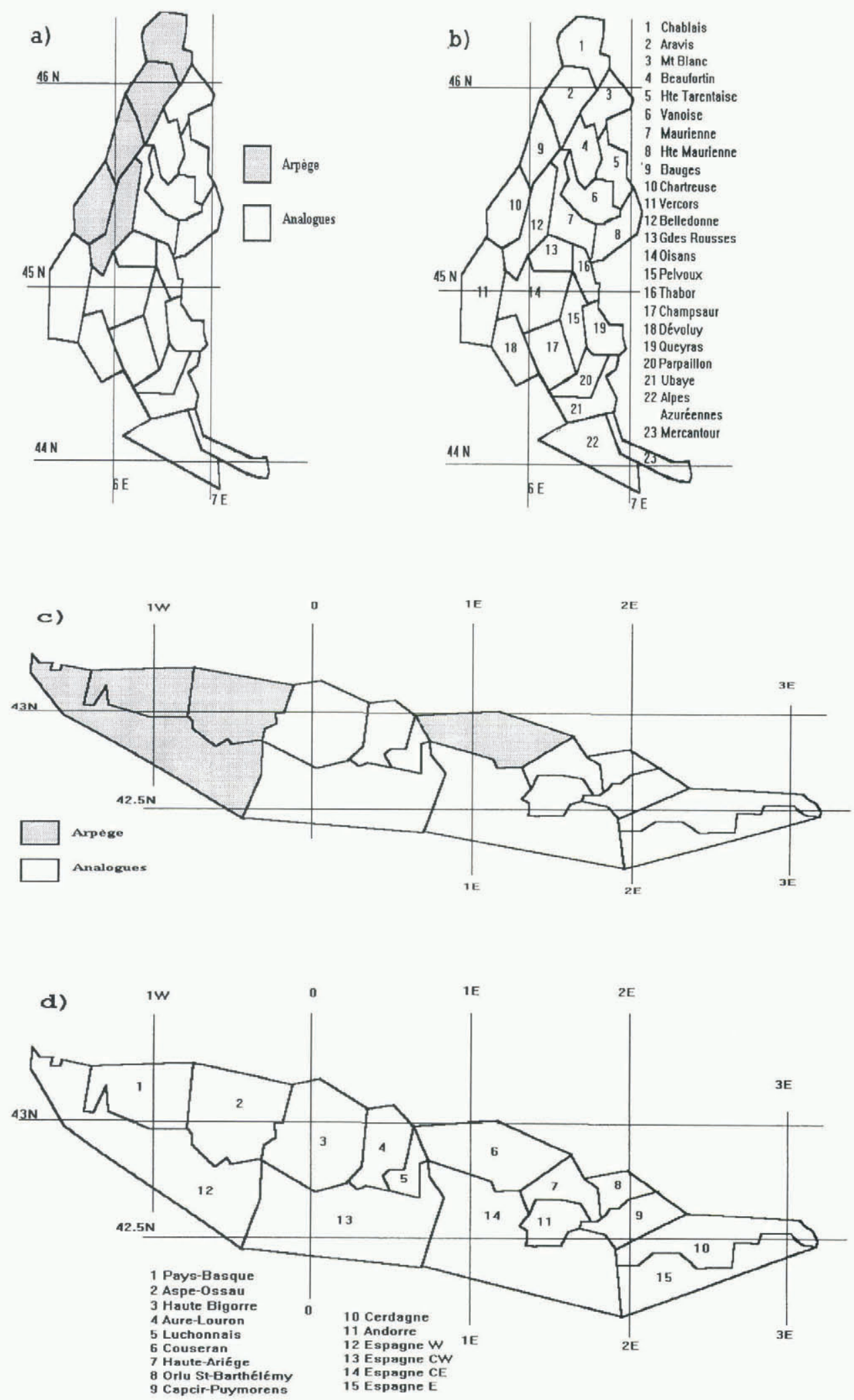

Fig. 4. Panels (a) (Alps) and (c) (Pyrenees) show the different massifs where the two methods (ARPĖGE or "analogue") provide, respectively, the best precipitation-forecast occurrence. We can therefore see the ARPEGE superiority in the northwest Alpine massifs ( $a$ ) and the western Pyrenean ones $(c)$, while the analogue method provides the best results elsewhere. This information is important for arbitrating on conflicts between the two methods. In addition, panels $(b)$ and $(d)$ list the names of the different massifs involved.

tion (816 cases), and in the Pyrenees, with a range of about 1 year (326 cases). This verification of the 24 hour forecasts was performed on all the massifs (23 in the Alps, 15 in the Pyrenees, cf. Fig. 4) and took as a reference the SAFRAN analysis (Durand and others, 1993) which runs the next day when real-time observations are available. This choice was debatable but convenient; the natural variability of the precipitation field in mountainous areas made direct comparison of forecasts and observations difficult, but the use of a reference analysis allowed comparison at the same scale (i.e. the massif, which is different from that observed) and at the same altitude $(1800 \mathrm{~m})$. It must be borne in mind, 

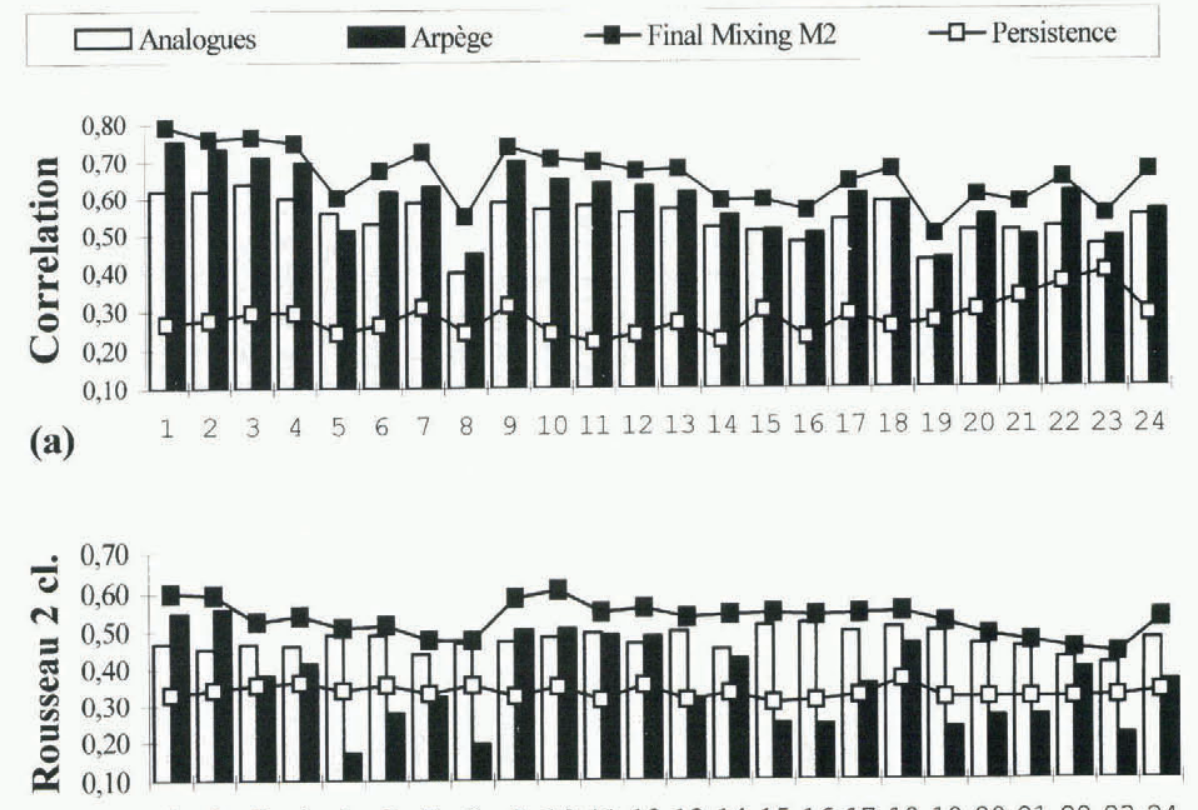

(b)

$\begin{array}{llllllllllllll}1 & 2 & 3 & 4 & 5 & 6 & 7 & 8 & 9 & 10 & 11 & 12 & 13141516171819202122 & 2324\end{array}$
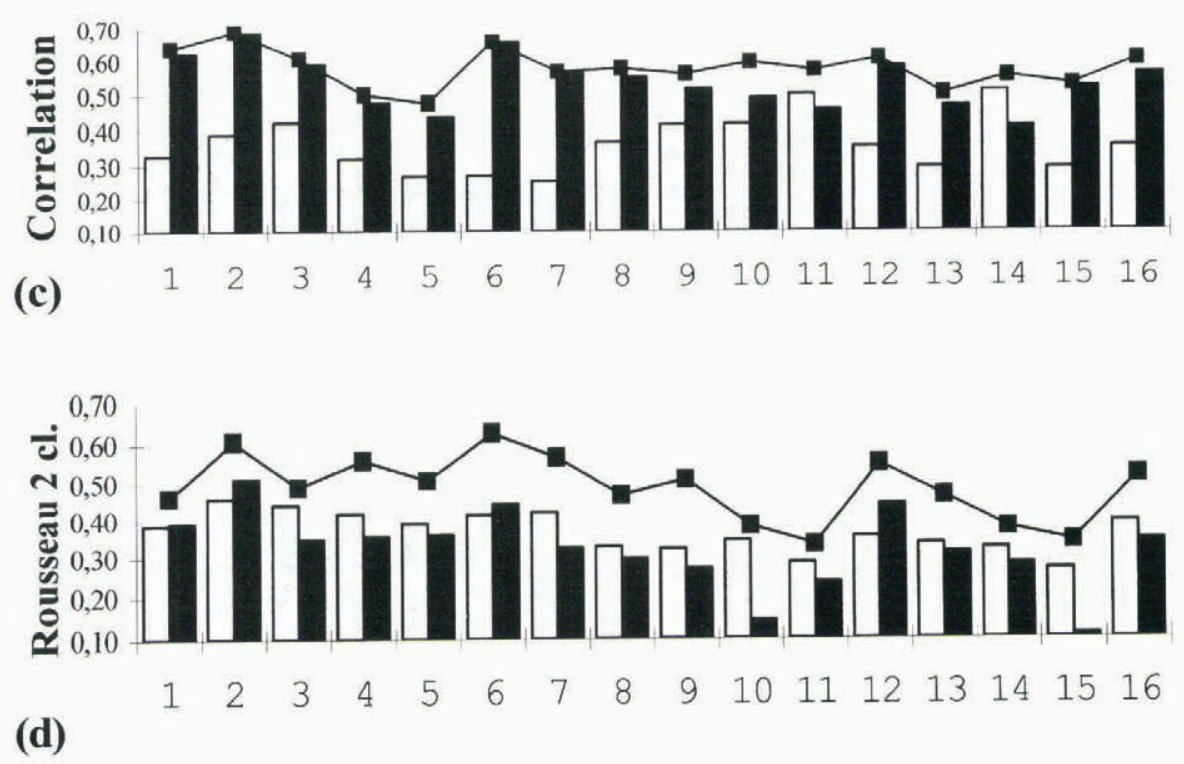

Fig. 5. Evaluation of the quality of 24 hour forecasted precipitation compared to reference analyses over 23 Alpine $(a, b)$ and 15 Pyrenean $(c, d)$ massifs ( see names in Figure 4). Plotted values are correlation $(a, c)$ and 2 class Rousseau score $(b, d)$ for different forecasting methods ("analogue", ARPEGE, "compound" mixing M2 and persistence (cf. text); all indicated on the top) vs analyses. The values plotted in $24(a, b)$ or in $16(c, d)$ on the $x$ axis correspond to an all-massif average.

however, that this reference field can be perturbed by different sources of error, especially during the off-winter season. This procedure can nevertheless be partially justified by different comparative studies at Col de Porte and given by Durand and others (1995).

The first comparisons, relating to the averaged precipitation values, exhibit a close similarity over the two areas between the reference analysis and the "analogue" forecast. We are confronted here with an artefact of our system. We are verifying that both analyses (reference running on the real date and analogue on the past date) have in fact the same internal climatology (according to seven typical weather conditions over the Alps and nine over the Pyrenees, cf. Durand and others, 1993). The mean value forecasted by ARPEGE is considerably different; it is much more important for the global average and is strongly influenced by the internal orography of the model. So, those oi.org/10.3189/1998AoG26-1-357-366 Published online by Cambridge University Press massifs with a high average height in the model (for instance, Haute Maurienne in the Alps or the Spanish massifs in the Pyrenees) are systematically overestimated in the $A R$ $P \grave{E} G E$ fields; this is, in fact, an indication of precipitation at a higher altitude than the reference one $(1800 \mathrm{~m})$. This first study allowed us still to compute debiased coefficients for every massif inside the ARPÉGE estimate with seasonal variability over the Alps. The Pyrenean coefficients were annual due to the small history interval of the learning file. All these coefficients are, of course, revised frequently when the size of the comparison dataset increases. The results are globally synthesized in Table 2 which shows daily averaged values over the Alps and Pyrenees; in addition some specific massifs are also given.

The second study was concerned with the correctness of the forecast of the "precipitation" phenomena for the following day, irrespective of its amount. This led to the study of a 
simple contingency table with two entries characterizing the occurrence of the phenomena. The verification index used is that of Rousseau (1980) which is well suited to precipitation and varies between 0 (very bad) to 1 (perfect). Unlike the classical indices (ratio test, Hansen and Kuiper index (Hansen and others, 1965) or the threat score), it is more constraining and systematically penalizes the biased forecasts. It retains the advantages of the different indices cited in being free of their disadvantages; in addition, its non-linear formulation is compatible with the definition of a distance between different sets. The results have finally shown that $A R P E G E$ forecasts were the best only in the Pré-Alps (northwest Alps, from Chablais to Chartreuse, cf. Fig.4) and also in the western Pyrenean massifs (Pays-Basque, Aspe-Ossau, cf. Fig. 4). This result agrees with the "classical" path of the synoptic perturbations reaching these massifs, which are well represented by the model. Nevertheless, the model seems to encounter some difficulty in representing the progression (or the blocking) of the precipitation areas inside the massifs, while the "analogue" method is obviously better for such cases. The results are synthesized in Table 2 which shows the global value of the two classes of Rousseau indices (denoted " $2 \mathrm{cl}$. Rousseau") over the areas, while Figure 5 (panels (b) and (d)) displays the spatial variability of this index according to the different Alpine (Fig. 5b) and Pyrenean (Fig. 5d) massifs. Figure 4 also summarizes these results by mapping the different massifs where the ARPEGE or "analogue" method provided the best forecast of rainfall occurrence. These results are not only validations but will be used later to arbitrate between cases where the forecasts of the two methods are different.

A third study, on another precipitation contingency table with five classes, basically confirmed the previous idea by showing the superiority of the ARPEGE distribution in the first western massifs, both in the Alps and the Pyrenees, while the "analogue" method provided best results everywhere else. The results can be seen in Table 2 (indicated " $5 \mathrm{cl}$. Rousseau") where the index used is a generalization of the previously cited Rousseau index described by Rousseau (1985).

We next studied the temporal correlation between the forecasted and analysed fields within each massif. The results are plotted in Figures $5 \mathrm{a}$ and $\mathrm{c}$ with the same previously described $x$ axis. Concerning these figures, we must note that the interpretation of the values presented is more difficult, because our reference field (the daily $S A F R A \mathcal{N}$ analysis) is inevitably slightly erroneous due to its own interpolation method or its use of false data or, more prosaically, the lack of observations from off-winter seasons. However, as mentioned previously, the main advantage is that the comparison-scale problems were reduced. Contrary to previous verifications, this study clearly favours ARPEGE; it demonstrates the best behaviour over most of the Alpine and Pyrenean massifs; its major weaknesses appears in the eastern Alpine massifs, e.g. Haute-Tarentaise. Here, the model poorly estimates the south and southwest meteorological flows and generates strong precipitation, where in reality it should be weak with a more-or-less pronounced föhn regime (personal communication from Bourg-St-Maurice forecasters). In the Pyrenees, only Andorra and a central Spanish massif are better treated by the "analogue" method than by $A R P E G E$. A brief synthesis of the results is also shown in Table 2 ; it illustrates the mean characteristics of the ARPE $G E$ adaptations (denoted by $\mathrm{P}$ ) and those of the analogue method $(\mathrm{S})$ by comparison with the analyses $(\mathrm{A})$. One should notice that these parameters can have strong annual variations as shown by the averaged Alpine correlations in Figure 6. From this figure, though limited to 3 years, we can appreciate the annual variations of the moving quarterly scores. The relatively bad summer scores are principally due to the convective characteristics of the rainfall which both methods have difficulty in representing, while the correlation value often exceeds 0.75 during the winter season. The contribution of each method is also evaluated with regard to the rough persistence method. This method which consists of taking the current analysed rainfall as the 1 day forecast for the following day is a convenient way of appreciating the quality of a forecasting method and the added value.

\section{MIXING OF ARPÈGE AND "ANALOGUE" METH- OD OUTPUTS}

Synthesis of the previous results, as well as of other studies (not presented here), which were carried out over shorter periods corresponding to different seasons with different rainfall conditions (frontal, convective and mixed), led us to mix the two solution procedures. The principle was to

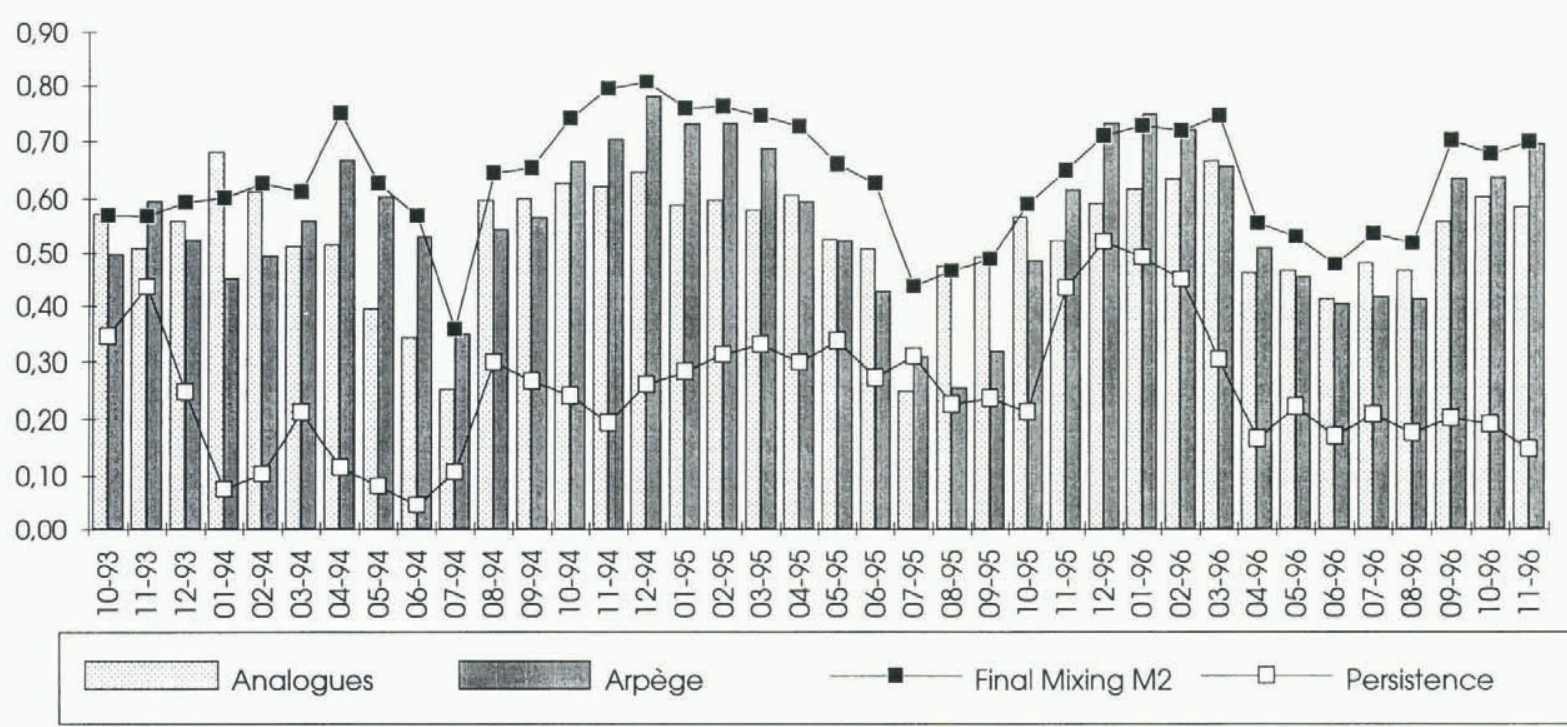

Fig. 6. Time variation of the correlation of 24 hour forecasted precipitation by different methods with the analysis. 
minimize the root mean square (rms) of the difference between the $S A F R A \mathcal{N}$ analysis and a linear combination of the two solutions given previously (the first statistically centred). The computation was done over 3 years for the Alps and 1 year for the Pyrenees. An immediate consequence was to achieve automatically a better correlation than with each of the components. This optimal mixing which takes information from each solution therefore provides a combination of better quality than each elementary solution (see Table 2, parameter Ml). The coefficients of the linear combination (hereafter denoted by $\alpha_{\mathrm{m}}$ ) are variable according to the massifs and are annual for the Pyrenees and seasonal for the Alps; explicitly, it is done as follows for each massif " $m$ " (the exponents " $s$ " and "p" indicate, respectively, "analogue" method results and ARPËGE forecasts):

$$
R_{\mathrm{m} 1}=\alpha_{\mathrm{m}} N_{\mathrm{m}}^{\mathrm{s}} R_{\mathrm{m}}^{\mathrm{s}}+\left(1-\alpha_{\mathrm{m}}\right) N_{\mathrm{m}}^{\mathrm{p}} R_{\mathrm{m}}^{\mathrm{p}}
$$

where $R_{\mathrm{m} 1}$ is a preliminary forecast result for given massif "m", and $R_{\mathrm{m}}^{\mathrm{s}}$ and $R_{\mathrm{m}}^{\mathrm{p}}$ are the "analogue" method and $A R$ $P E G E$ forecasts for " $m$ " massif, $\alpha_{\mathrm{m}}$ is the combination coefficient between the two methods (see below), and $N_{\mathrm{m}}^{\mathrm{s}}$ and $N_{\mathrm{m}}^{\mathrm{p}}$ are normalization factors (see below).

The combination coefficients are in the range of $0.2-1.5$ and are regularly updated according to the rms minimization criteria (see above) which is applied in every massif. They show (not presented here) that in each massif the mixing gives the advantage to ARPEGE; more pronounced in the Pyrenees than in the Alps (which can be seen in Figure $5 c$ ). The forecasted quantities are also renormalized according to the operational SAFRAN analysis; this operation is much more important for the ARPE GE fields (as explained in the previous paragraphs; see Table 2, field P). We can therefore interpret this mixing as the result of a large-scale field, which is renormalized by massif and in phase with the meteorological reality, weighted by analogue-method outputs which are intrinsically less efficient but are able to carry small-scale information both with regard to the occurrence of the event "precipitation" and the variability between massifs.

The emerging $R_{\mathrm{m} 1}$ is successful in terms of the correlation with the analyses. The use of seasonal coefficients provides good quality in wintertime when the reference analyses are reliable. On the other hand, this mixing exhibits totally deplorable contingency characteristics (cf. previous section) in the two or five Rousseau cases (cf. Table 2). The explanation is simple; the wrong forecasts for each of the two components of the mixing were accumulative. So, it is sufficient for one of the two to produce a wrong "rainy" forecast to obtain immediately an erroneous mixing solution. On the other hand, two "dry" components are needed to make a successful dry forecast. All these problems are synthesized by a drastic decrease of the Rousseau score when it is applied to the contingency tables produced by this preliminary mixing.

An improvement of this first intermediate result was therefore necessary and has been achieved through an algorithm whose purpose is to solve the case where, in a particular massif, one method gives a rainy result and the other gives a dry result. The adjustment process is based on the two Rousseau scores which have indicated (cf. previous sections and Figure 4) the respective massifs where each method is better for forecasting the occurrence of precipitation. The optimal solution is therefore to take only the most suitable method (according to Rousseau) in every massif (without mixing) when the two estimates are different in their forecasting of the occurrence of precipitation. The best-averaged $A R P E G E$ quality is still present in the algorithm; as a matter of fact, a strong precipitation ARPÉGE forecast is retained even in massifs where one should take a dry analogue-method forecast according to the previous criteria. All these rules are applied in both the Alps and the Pyrenees, and are used to compute a final field $R_{\mathrm{m} 2}$ which has been proven to be better than the other estimates for all the previously mentioned statistical tests (mean, correlation and Rousseau) as shown in Table 2 and Figures 5 and 6 (and denoted by M2).

\section{VALIDATION BY COMPARISON BETWEEN MÉPRA-ESTIMATED AND FORECASTED RISKS}

In order to evaluate the quality loss of this "forecast chain" with regard to the "analysis chain" results and to complete the SAFRAN $N$ tests, qualitative comparisons were done. We compared MÉPRA natural risks coming from both the "analysis" and the "forecast chain" that are valid for the same day. To carry out these comparisons, the measurement of MÉPRA risks was defined. Different indices were tested and one was chosen that summarized the great spatial variability and intensity of the MÉPRA natural risks within a massif. To account for the intensity of the risk, each level of the MÉPRA scale was assigned a weighting of 0 for very low and low risk, 2 for moderately decreasing risk, 3 for moderately increasing risk, 4 or 5 for high or very high risk. The MÉPRA index is, in fact, the average of the MÉPRA natural risk between the elevations of 1500 and $3000 \mathrm{~m}$ for all aspects (north, east, southeast, south, southwest and west). In the past, the same index was also used to compare naturally observed avalanche activity with MÉPRA risks (Giraud and others, 1994). For this validation, 5 years of comparisons were done for the Vanoise massif in the Savoy department. A small decrease in quality was measured except when forecast errors concerned the precipitation values. The 1994-95 winter example (Fig. 7, panels (a) and (b)) shows three periods with high forecasted risks and very low estimated risks, 22 December 1994, 4 and 29 March 1995. These spectacular errors are due to wrong forecasts of precipitation values, near $40-50 \mathrm{~cm}$ of forecast fresh snow even though no precipitation was observed. In the other cases, the results are quite good and the forecast risks overlap the estimated risks.

These numerical results can be compared (Fig. 7, panel (c)) with an observed avalanche-activity score deduced from the daily data which are sent by the snow patrolers at the ski resorts. This score is expressed on a different scale of about 16 levels (Giraud and others, 1987) and shows good agreement with previous results (Fig. 7, panels (a) and (b)), especially in terms of phasing. Nevertheless, the difficulty of observing all the avalanche events (especially in bad weather conditions) makes quantitative comparisons difficult to achieve.

\section{GONCLUSION}

Meteorological and avalanche forecasts in mountainous areas are difficult exercises from which one still has a lot to learn. Our attempt, though still based on a too-limited series of comparisons, is a appropriate answer to the demands for a local "mountainous forecasting" dealing with smallscale phenomena. We have presented a solution, which uses the different existing tools and provides a 24 hour forecast 
(a)

\section{Daily integrated Mépra risks from the Analysis Suite}

(Vanoise, winter season 1994-95)

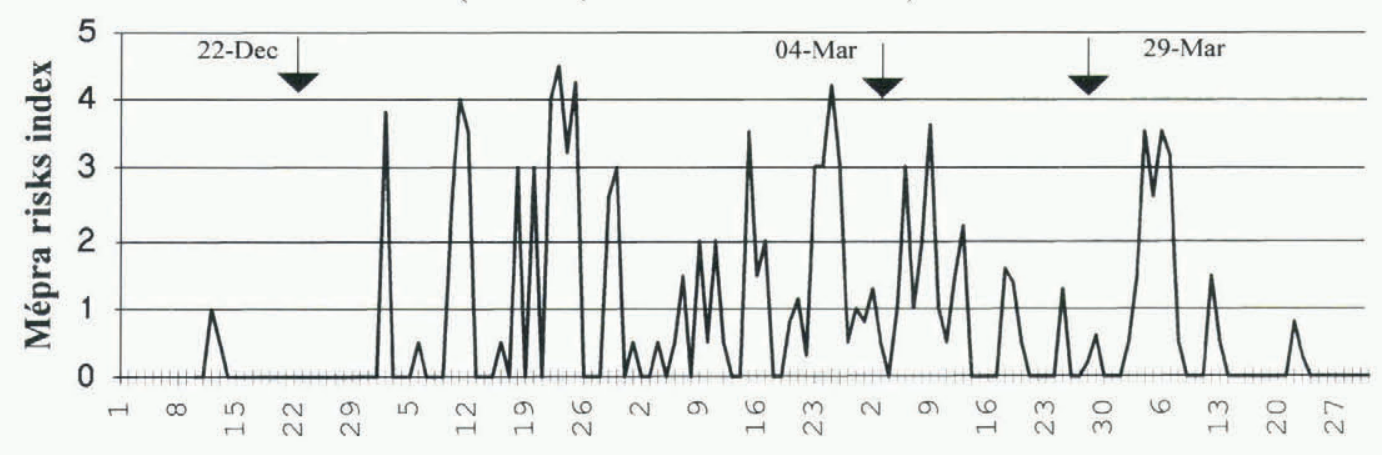

(b) Daily integrated Mépra risks from the Forecasting Suite

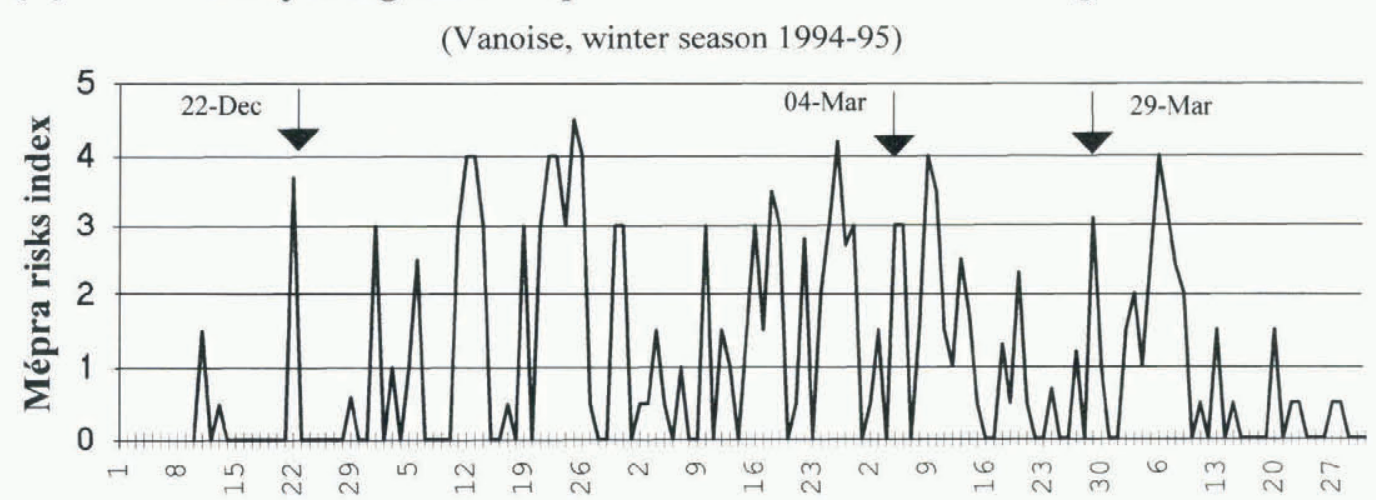

(c)

Observed Avalanche Score over Vanoise Massif

(Season 1994-95)

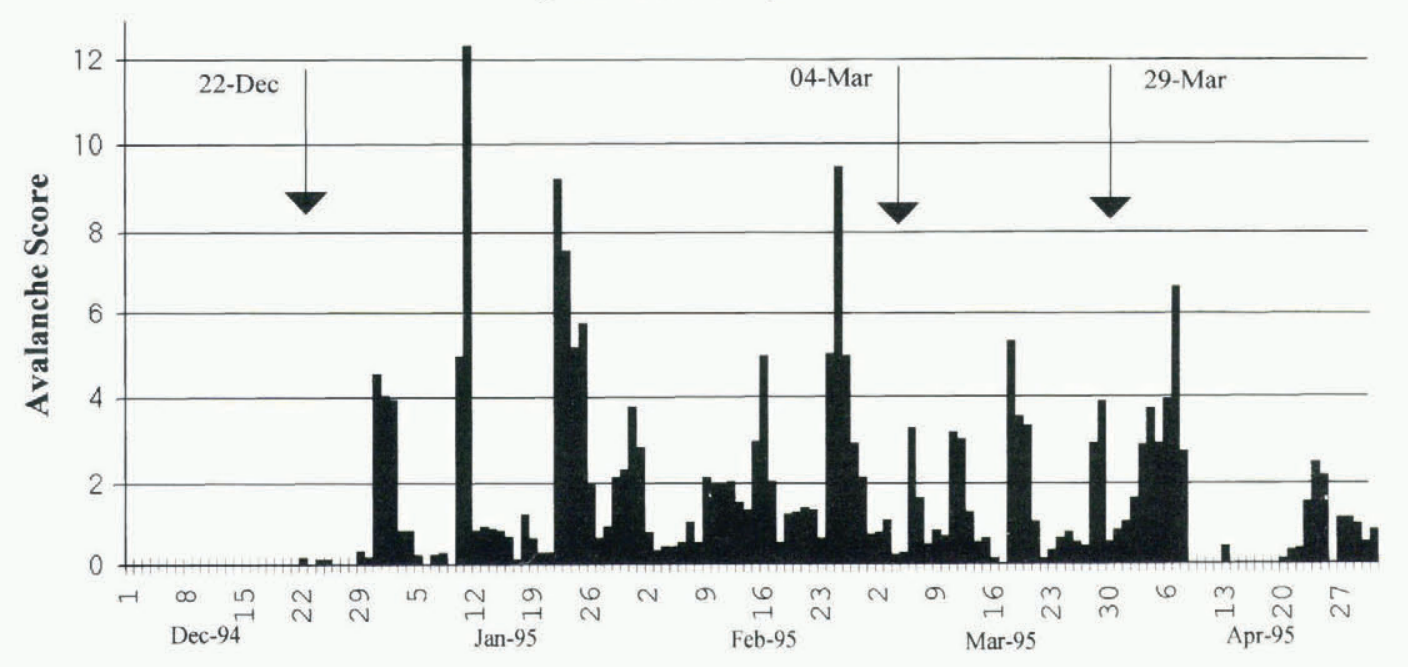

Fig. 7. Comparison between MÉPRA estimated risks (a) and MÉPRA forecast risks (b) (plotted on a five-level scale) and verification with an observed avalanche score $(c)$ (plotted on a different scale of about 16 levels). The three panels cover the same winter season 1994-95 (same $x$ axis) in the Vanoise massif.

for the different meteorological parameters that are relevant for numerical snow simulation and related avalanche risks. It is based on down-scaling the larger-scale ARPE $G E$ fields and using analogous situations from the past. If, for all quantities except precipitation, the down-scaling method provides the best results, the precipitation forecast is the result of a combination of the two previous methods. The impact of the "analogue"-method forecast is at once "sensible" to the forecasters, who appreciate obtaining an earlier situation, and is especially discriminating for the precipitation-forecast occurrence. $A R P E G E$ gives valuable averaged information and mixing only increases the quality of the forecast of precipitation produced. This mixing must still be automatically supervised in order to avoid wrong fore- 
casts of each component. The system is now used in realtime by forecasters as a supplement to traditional tools and provides a full snowpack description covering the whole range of avalanche reports.

\section{ACKNOWLEDGEMENTS}

All these models needed many observational data which were daily provided by observers in different networks, especially by those from the snow patrols at ski resorts. We are most grateful for their work.

We also thank the scientific editor and the reviewers for their useful comments and helpful suggestions.

\section{REFERENCES}

Brun, E., E. Martin, V. Simon, C. Gendre and C. Coléou. 1989. An energy and mass model of snow cover suitable for operational avalanche forecasting. 7. Glaciol., 35(121), 333-342.

Brun, E., P. David, M. Sudul and G. Brunot. 1992. A numerical model to simulate snow-cover stratigraphy for operational avalanche forecasting. 7. Glaciol., 38(128), 13-22.

Courtier, P., C. Freydier, J.-F. Geleyn, F. Rabier and M. Rochas. 1991. The Arpège project at Météo-France. ECMWF Seminar Proceedings, September 9 13 1991, Reading, U.K.Vol. 2. Reading, U.K. European Centre for MediumRange Forecasts, 193-232.

Duband, D. 1981. Prévision spatiale des hauteurs de précipitations journalières. Houille Blanche, juillet-août, 497-511.

Durand, Y., E. Brun, L. Mérindol, G. Guyomarc'h, B. Lesaffre and E. Martin. 1993. A meteorological estimation of relevant parameters for snow models. Ann. Glaciol., 18, 65-71.
Durand,Y., L. Mérindol and S. Michoud. 1995. Premiers éléments pour une prévision numérique du risque d'avalanche au cours de la journée du lendemain. In Sivardière, F., ed. Les apports de la recherche scientifique à la sécurité neige, glace et avalanche. Actes de Colloque, Chamonix 30 mai-3 juin 1995. Grenoble, Association Nationale pour l'Étude de la Neige et des Avalanches (ANENA), 169-175.

European Centre for Medium-Range Forecasts (ECMWF). 1991. MARS user guide. Reading, U.K. European Centre for Medium-Range Forecasts. (ECMWF Computer Bulletin B6.7(2).)

Giraud, G. 1993. MEPRA: an expert system for avalanche risk forecasting. In Armstrong, R., ed. ISSW'92. A Merging of Theory and Practice. International Snow Science Workshop, 4-8 October 1992, Breckenridge, Colorado. Proceedings. Denver, CO, Avalanche Information Center, 97-104.

Giraud, G., J. Lafeuille and E. Pahaut. 1987. Évaluation de la qualité de la prévision du risque d'avalanche. International Association of Hydrological Sciences Publication 162 (Symposium at Davos 1986-Avalanche Formation, Movement and Effects), 583-591.

Giraud, G., E. Brun, Y. Durand and E. Martin. 1994. Validation of objective models to simulate snow cover stratigraphy and avalanche risks. In ISSW'94. International Snow Science Workshop, 30 October - 3. November 1994, Snowbird, Utah. Proceedings. Snowbird, UT, P.O. Box 49, 509-517.

Hansen, A. W. and W.J. A. Kuipers. 1965. On the relationship between the frequency of rain and various meteorological parameters. Koninklijk Nederlands Meteorologisch Instituut. Mededelingen en Verhandelingen 81, 2-15.

Navarre, J. P. 1980. Prévision des précipitations sur les Alpes par une analyse des situations analogues. In 16ième Congrès International de Météorologie Alpine, Septembre 1980, Aix-les-Bains, France. Comptes-rendus. Toulouse, MétéoFrance, 209-213.

Rousseau, D. 1980. A new skill score for the evaluation of yes/no forecasts. In WMO Symposium on Probabilistic and Statistical Methods in Weather Forecasting, September 1980, Nice. Geneva, World Meteorological Organization, $167-174$.

Rousseau, D. 1985. Compléments sur un critère de vérification de prévisions catégoriques. Toulouse, Météo-France. Établissement d'Études et de Recherches Météorologiques. (Note de Travail 123.) 